\title{
植物激素定量分析方法研究进展
}

\author{
符继红 ${ }^{(12)}$ ，孙晓红 ${ }^{(2)}$ 王吉德 ${ }^{(1)}$ 褚金芳 ${ }^{(2 *}$ ，间存玉 ${ }^{(2 *}$ \\ (1) 新疆大学化学化工学院, 乌鲁木齐 830046 ; \\ (2) 中国科学院遗传与发育生物学研究所, 国家植物基因研究中心(北京), 北京 100101 \\ * 联系人, E-mail: cunyuyan@genetics.ac.cn; jfchu@genetics.ac.cn
}

2010-07-13 收稿, 2010-09-13 接受

国家自然科学基金资助项目(90917017,90817008)

\begin{abstract}
摘要 植物激素是对植物生长发育具有重要调控作用的小分子化合物, 在低浓度下就 能发挥生理作用, 参与调控植物生长发育的每一过程. 植物激素的合成、运输、代谢和 分子作用机理的深入研究都需要对植物激素进行定量分析. 但是, 植物激素定量分析 受到低含量、次生代谢产物背景千扰严重等因素的影响, 一直是植物激素研究领域的瓶 颈。近年来，随着植物激素在提取、纯化和检测方法等方面的发展，植物激素定量分析 取得一定进展. 固相萃取技术和色谱/质谱联用技术的发展为植物激素的高效提取纯化 和准确定量分析提供了可能, 成为植物激素定量分析领域广泛被接受的技术手段. 此 外, 液相萃取、免疫纯化、免疫分析和电化学分析等纯化检测方法在植物激素分析中也 有应用, 本文对各种纯化检测方法进行了比较和讨论. 随着植物激素调控机理和植物 激素互作研究的深入, 需要对原位、动态和多种植物激素同时检测, 这将是植物激素分 析领域的未来研究方向.
\end{abstract}

关键词

植物激素

提取方法

液相萃取

固相萃取

免疫纯化

免疫分析

电化学分析

色谱及色谱质谱联用
植物激素是植物体内合成的对植物生长发育有 显著作用的微量有机物, 能从产生部位转移到作用 部位, 在低浓度下就能调节植物的生长发育, 几乎参 与了调控植物生长发育的每一过程, 从影响细胞的 分裂、伸长、分化到影响植物发芽、生根、开花、结 实、休眠和脱落等 ${ }^{[1]}$, 主要包括乙烯(ethylene)、生长 素 (auxin)、脱落酸 (abscisic acid)、赤霉素 (gibberellins)、细胞分裂素(cytokinins)、茉莉酸(jasmonates)、 油菜素内酯(brassinosteroids)和水杨酸(salicylates)等 (表 1). 另外, 一些次生代谢产物, 如一氧化氮(NO) 及近年来发现的独角金内酯(strigolactones)等也被认 为在调节植物生长发育方面以类似激素的方式起作 用. 当植物受到病原体侵染、害虫及动物的啃咬、干 旱及盐胁迫等生物和非生物的伤害后, 植物激素在 植物启动防御反应的过程中也发挥着巨大的作 用 ${ }^{[2 \sim 4]}$. 植物激素既可以通过调控作物生长发育等过
程而直接影响作物产量, 又可以通过参与调控作物 对各种不利条件的适应性而减少产量损失, 其研究 成果对提高作物产量起到了革命性的推动作用, 具 有较大的农业应用价值, 因而植物激素在植物体内 含量的准确测定越来越引起人们的重视. 植物体内 的激素含量极低, 性质不稳定, 容易受到其他次生代 谢产物的干扰; 同时, 部分用于植物生理学研究的植 物突变体材料非常珍贵, 材料量少, 因此要求检测的 方法必须十分灵敏和专一. 国家自然科学基金委员 会已经就植物激素研究设立了重大研究计划项目, 其中一个核心科学问题就是解决植物激素超微定量 检测的技术瓶颈.

植物激素的提取纯化与检测通常包括以下几个 关键的步骤: 首先, 在样品的收集过程中, 植物材料 应迅速用液氮冷冻, 以保证所分析的样品最大限度 保持植物的原始状态, 没有因为植物受伤而导致植 
表 1 主要植物激素及代表性化合物的分子结构和名称

\begin{tabular}{|c|c|c|}
\hline \multirow{2}{*}{ 类别 } & \multicolumn{2}{|c|}{ 代表性植物激素 } \\
\hline & 结构 & 名称 \\
\hline Auxins & & Indole-3-acetic acid (IAA) \\
\hline Abscisic acid (ABA) & & S-(+)-Abscisic acid (S-ABA) \\
\hline Jasmonates (JAs) & & $(-)$-Jasmonic acid $((-)-J A)$ \\
\hline Gibberellins (GAs) & & Gibberellin $\mathrm{A}_{1}\left(\mathrm{GA}_{1}\right)$ \\
\hline Salicylates (SAs) & & Salicylic acid (SA) \\
\hline Cytokinins (CKs) & & trans-Zeatin $(\mathrm{Z})$ \\
\hline Ethylene & C & Ethylene \\
\hline Strigolactones (SLs) & & 2'-epi-5-Deoxylstrigol (epi-5DS) \\
\hline Brassinosteroids (BRs) & & Brassinolide (BR) \\
\hline
\end{tabular}

物激素含量的失真, 并在液氮保护下研磨成粉末; 其 次，选择合适的提取溶剂对目标激素进行高效率的 提取; 再次, 优化纯化方法, 对植物粗提物进行纯化 和富集; 最后, 建立灵敏的测定方法, 对植物样品中 的植物激素进行准确可靠的分析. 因此高选择性的
样品提取纯化方法和高灵敏的测定技术的开发将是 植物激素检测的研究重点. 植物激素提取与检测技 术的快速发展为研究激素在代谢、信号转导及互作网 络机理等方面提供了直接的量化信息，从而为进一 步阐明植物激素的作用机理提供帮助. 


\section{1 提取与纯化方法}

植物激素作为特殊的植物次生代谢产物群体, 在植物体内的含量非常低, 通常只有普通植物次生 代谢产物的万分之一甚至更低, 在 $0.1 \sim 50 \mathrm{ng} \cdot \mathrm{g}^{-1}$ 鲜 重范围内 ${ }^{[5]}$. 同时, 植物提取物又是复杂的多组分混 合物, 其所含的次生代谢产物会对植物激素的定性 和定量分析产生严重的干扰. 因此, 为鉴别和定量测 定植物激素, 必需建立和优化植物激素的提取和纯 化流程, 尽量除去植物粗提物中影响植物激素分析 的杂质, 富集目标化合物, 以得到充分纯化的待分析 样品供最终分析测定.

\section{1 提取方法}

植物激素分析首要工作是从植物材料中将目标 植物激素充分提取出来. 这取决于两个方面: 一是植 物材料的处理, 通常选择液氮冷冻条件下的新鲜或 冻干处理的植物材料进行充分研磨; 二是选择合适 的提取方法将植物激素从这些材料中提取出来.

目前, 溶剂提取法仍然是植物激素研究采用的 最广泛的提取方法. 甲醇、丙酮、丙醇及其水溶液和 中性、酸性缓冲溶液是较为常用的提取液 ${ }^{[6 ~ 13]}$, 有机 溶剂与水的比例通常需要依据被检测植物激素的极 性来确定, 极性很小的溶剂如乙醚通常不用来提取 植物激素. 在这些有机溶剂体系中, 甲醇由于分子量 小, 渗透性较强, 在提取过程中可以渗透到细胞中, 一直是植物激素提取的首选溶剂, 被广泛地用于植 物激素的提取 ${ }^{[14 ~ 23]}$. 在提取过程中, 部分植物激素 化学性质不稳定, 容易受到提取环境的影响. 例如, 生长素类植物激素容易氧化或见光分解，在分析提 取过程中一定要避免光照、高氧和高温等外部环境, 因此需要在提取过程中加人抗坏血酸、铜试剂等电极 电位较低的抗氧化剂, 保证其在提取过程中的稳定. 除了外部环境对部分目标植物激素稳定性的影响之 外, 一些植物激素在提取过程中还会受到植物体内 酶的影响. 例如, 在细胞分裂素的提取过程中, 植物 体内的碱性磷酸酯酶会造成核苷酸型细胞分裂素的 水解, 这种酶在 $80 \%$ 甲醇提取液中仍保持一定活性, 采用该溶剂体系会对分析结果产生不利影响，改进 的方法是使用 Bieleski 试剂 $\left(\mathrm{MeOH}: \mathrm{CHCl}_{3}: \mathrm{HCOOH}\right.$ : $\mathrm{H}_{2} \mathrm{O}=12: 5: 1: 2$, 体积比), 它能有效降低核苷酸型细 胞分裂素酶解为核苷 ${ }^{[24]}$. 但该提取液极性偏小, 会
将植物组织中的大量脂类物质提取出来，对后续细 胞分裂素的进一步纯化造成困难，不利于细胞分裂 素的定量分析 ${ }^{[25,26]}$. 在此基础上, 通过对有机溶剂和 配比进行调整, 改良型 Bieleski 试剂 $(\mathrm{MeOH}: \mathrm{HCOOH}$ : $\mathrm{H}_{2} \mathrm{O}=15: 1: 4$, 体积比)被应用于细胞分裂素和赤震素 等多种植物激素的提取 ${ }^{[27,28]}$. 尽管改良型 Bieleski 试 剂能够减少脂类物质的溶出，简化后续的纯化富集 处理流程，但在酸性条件下，细胞分裂素核苷会发生 降解释放出游离的细胞分裂素, 对定量分析结果也 会产生影响.

所以，提取溶剂的选择主要取决于目标植物激 素的物理化学性质和植物样品的性质, 并综合考虑 提取过程中提取环境和植物激素在植物体内存在的 形态等影响因素, 其基本原则是在不改变植物激素 成分变化条件下, 最大限度地提取出目标植物激素 并尽量降低干扰杂质的溶出.

\section{2 纯化方法}

一般而言, 通过有机溶剂提取的植物粗提物中 含有大量的植物次生代谢产物，而植物激素在粗提 物中含量极低, 这些植物次生代谢物会对植物激素 的定量分析产生很强的干扰，影响分析结果的准确 性, 因此植物粗提物并不适合直接进行植物激素定 量分析. 通过对植物粗提物进行一系列的后续处理, 能够将感兴趣的目标植物激素进行纯化和富集，降 低或者消除植物组织中大量复杂的背景干扰，从而 使样品适合定量分析. 随着对植物激素研究工作的 深人, 从经典的液相萃取到固相提取越来越多的新 技术和新方法被引人到植物激素分析领域.

(i ) 液相萃取. 液相萃取主要是利用目标化合 物组分在互不相溶的溶剂体系中溶解度的不同而达 到分离或纯化目的. 对于一些植物组织中含量相对 较高的植物激素, 利用液相萃取法对植物提取物进 行纯化处理, 也能得到较好的分析结果. 在实际实验 中，可根据植物激素的物理化学性质，选择乙酸乙 酯、二氯甲烷、乙醚等有机溶剂纯化植物样品 ${ }^{[7,9,15]}$. 但是, 传统液相萃取一般需要经历较多萃取步骤，且 消耗时间较长，同时还要使用较多的有机溶剂.值得 注意的是，液相萃取技术，对水溶性较好的植物激素 纯化效率不高, 并且提取过程中还会产生难以克服 的乳化现象，这些缺点限制了液相萃取法在微量植 物激素提取中的应用，目前在实验室中已较少使用. 
最近, 有研究人员通过对传统液相萃取技术的改进, 开发了基于微孔径的纤维管的液-液-液微萃取方法, 其基本原理是将目标分析物从水中萃取到有机相, 再从有机相萃取到第二个水相, 通过调节样品和萃 取相的 $\mathrm{pH}$ 完成液-液-液的萃取富集过程, 能够对椰 子汁中的 IAA, ABA, SA 和 JA 等 4 种酸性植物激素 同时提取纯化 ${ }^{[29]}$. 尽管该方法溶剂用量较少具有快 速、低消耗的优点, 但是液-液-液萃取过程中有机相 的选择要遵循和水互不相溶、与微孔纤维管有相近的 极性及目标化合物在有机相中的溶解性高于样品溶 液又低于最后的萃取水相等原则, 其体系的设计和 选择性限制了该技术的广泛应用. 因此, 对于植物激 素分析工作, 液相萃取只能部分解决植物激素分析 中的纯化富集问题, 远远不能满足现代科研工作者 对样品处理过程的通用、快捷、方便和高通量的分析 要求.

(ii) 固相萃取. 固相萃取 $(\mathrm{SPE})$ 技术是一个包 括液相和固相的物理萃取过程, 其主要目的是把痕 量被测定组分进行浓缩和富集. 固相萃取柱用以保 留目标化合物, 并尽量减少干扰杂质的保留, 选择合 适的溶剂将干扰杂质淋洗掉, 然后再用另一溶剂把 感兴趣的分析物从固定相上洗脱下来; 反之, 也可让 目标化合物直接通过固定相而不被保留, 同时大部 分干扰物质被保留在固定相上, 从而得到分离. 与传 统的液相萃取技术相比, 固相萃取不需要大量互不 相溶的有机溶剂, 可供选择的固定相种类较多, 具有 快速、可靠、消耗试剂少、易于实现自动化等优点, 能 够对复杂基质样品中的目标化合物进行纯化和富集, 满足了人们对处理方法的高效、快捷、简单和低消耗 的要求, 被广泛用于植物激素类样品的处理. 固相萃 取操作要充分利用目标植物激素的物理化学性质, 例如酸碱性和极性等, 选择合适的固相萃取材料和 处理流程，达到目标植物激素纯化富集的目的.

在对植物样品中植物激素的提取工作中, 有实 验室利用高效液相色谱(HPLC)馏分收集用于植物激 素的纯化富集, HPLC 也可理解为一种特殊的固相萃 取技术手段, 粗内径的凝胶柱、 $\mathrm{C}_{18}$ 柱和 ODS 柱都曾 被用于植物激素的纯化 ${ }^{[30 ~ 32]}$. HPLC 馏分收集法能够 高效地富集目标植物激素, 大幅度除去背景干扰, 使 测定结果更为准确可靠. 但 HPLC 纯化法需要较长时 间, 所使用的色谱柱价格也较为昂贵, 并消耗大量的 有机溶剂, 因此 HPLC 纯化法现在已较少使用, 仅在
部分植物体内含量极低的植物激素(如赤霉素等)定 量分析中还在使用 ${ }^{[33]}$.

与 HPLC 馏分收集富集纯化植物激素技术相比, 固相萃取技术在植物激素研究中的应用日益广泛, 这种技术简化了固相萃取流程，样品处理时间大大 缩短, 并且具有很好的重现性. 较为常用的固相萃取 小柱(表 2 )包括离子交换柱 ${ }^{[34,35]}$ 、反相色谱柱 $\left(\mathrm{C}_{18}\right.$ 柱 ${ }^{[16,36 ~ 40]}$ 、Oasis HLB 柱 $^{[8,28,41]}$ )、混合模式色谱柱 (Oasis MAX 柱 ${ }^{[41]}$ 、Oasis MCX 柱 ${ }^{[28,39,40]}$ )等, 在分析 样品时由于样品基质的复杂性有时需将几种方法结 合使用. $\mathrm{C}_{18}$ 柱是一种反相机理保留的固相柱, 也是 使用最为广泛的一种样品处理小柱. 将过 $\mathrm{C}_{18}$ 柱前的 溶液调成弱酸性, 抑制了具有酸性的植物激素的电 离而能更好地保留在反相 $\mathrm{C}_{18}$ 柱上, 最后用相应的甲 醇或乙醇的酸性水溶液(一般用甲酸或乙酸调节酸 度)将目标化合物洗脱下来. $\mathrm{C}_{18}$ 可以较好地除去植物 组织中的部分色素和极性化合物. 但 Hou 等人 ${ }^{[43]}$ 发 现在最后的洗脱步骤中不加酸的甲醇比加了少量酸 ( $1 \%$ 甲酸)的甲醇具有更好的洗脱效果. 作者认为在 酸性甲醇中植物激素的电中性在反相 $\mathrm{C}_{18}$ 上更强, 所 以更难洗脱.

在植物激素分析过程中, 通常会根据植物样品 的复杂程度、目标植物激素的物理化学性质和固相萃 取柱的特点, 联合使用两种或者两种以上固相萃取 柱对植物提取物进行处理, 富集纯化目标植物激素. 阴离子交换柱和阳离子交换柱分别可以较好地保留 植物提取物中的酸性化合物和碱性化合物, 通过合 适的预柱平衡处理和调节上样样品的 $\mathrm{pH}$, 使得相应 植物激素电离成相应的阴离子或阳离子而被很好的 保留, 再用相应的酸性或碱性甲醇溶液将目标化合 物洗脱下来, 因此在固相萃取柱的联合使用中这两 种 $\mathrm{SPE}^{[27,40,41]}$ 柱常被选择. 细胞分裂素为一类化学性 质特殊的化合物, 可以酸碱两性电离, 其嘌呤环上 6 位碳上连接的 $\mathrm{N}$ 原子呈碱性, $\mathrm{p} K_{\mathrm{b}} \approx 4$, 咪唑环上 9 位 $\mathrm{N}$ 原子呈弱酸性, $\mathrm{p} K_{\mathrm{a}} \approx 10$, 当改变溶液 $\mathrm{pH}$ 时, 所有 的细胞分裂素都可以电离成相应的正负离子 ${ }^{[25]}$. 而 植物内源性干扰物细胞分裂素核苷酸则由于带有 1 个磷酸基团而具有 2 个酸性电离常数 $\mathrm{p} K_{\mathrm{a} 1}(\approx 1.0)$ 和 $\mathrm{p} K_{\mathrm{a} 2}(\approx 6.0)$, 其化学特性为细胞分裂素的分离纯化提 供了极大帮助. $\mathrm{Ge}$ 等人 ${ }^{[40]}$ 在提取椰汁中的细胞分裂 素时首先采用 $\mathrm{C}_{18}$ 柱除去样品中的脂类物质和部分色 素，随后采用反相阳离子柱 Oasis $\mathrm{MCX}$ 进一步纯化 
表 2 常见固相萃取柱的特性及其在植物激素分析中的应用

\begin{tabular}{|c|c|c|c|c|c|c|}
\hline SPE 名称 & 分类 & 基本原理 & 分析物性质 & $\mathrm{pH}$ & 分析物 & 参考文献 \\
\hline DEAE-cellulose & 阴离子交换 & $\begin{array}{c}\text { 以纤维素为基质键合二乙胺 } \\
\text { 基乙基具有阴离子交换保留 } \\
\text { 机理 }\end{array}$ & 阴离子/中极性 & $2 \sim 9.5$ & CTKs & [34] \\
\hline SCX & 阳离子交换 & $\begin{array}{c}\text { 以硅胶为基质键合苯磺酸基 } \\
\text { 具有阳离子交换保留机理 }\end{array}$ & 阳离子/非极性 & $2 \sim 8$ & CTKs & [35] \\
\hline $\mathrm{C}_{18}$ & 反相 & $\begin{array}{c}\text { 以硅胶为基质键合十八烷基 } \\
\text { 具有反相保留机理 }\end{array}$ & 非极性/弱极性 & $2 \sim 7.5$ & $\begin{array}{l}\text { JA, ABA, IAA, } \\
\text { IBA, GA, Z, BA, } \\
\text { KT, CTKs }\end{array}$ & {$[16,36 \sim 40]$} \\
\hline Oasis HLB & 反相 & $\begin{array}{c}\text { 由亲脂的二乙烯苯和亲水的 } \\
\mathrm{N}-乙 \text { 乙烯基吡咯烷酮两种单体 } \\
\text { 按一定比例聚合而成具有反 } \\
\text { 相保留机理 }\end{array}$ & 非极性/弱极性 & $1 \sim 14$ & $\begin{array}{c}\text { IAA, ABA, GA, } \\
\text { CTKs }\end{array}$ & {$[8,28,41]$} \\
\hline Oasis MAX & $\begin{array}{c}\text { 阴离子交换-反 } \\
\text { 相混合模式 }\end{array}$ & $\begin{array}{c}\text { 以高聚物苯乙烯/二乙烯苯为 } \\
\text { 基质键合季铵基团具有阴离 } \\
\text { 子交换和反相吸附两种保留 } \\
\text { 机理 }\end{array}$ & 阴离子/非极性 & $1 \sim 14$ & $\begin{array}{l}\text { IAA, ABA, } \\
\text { CTKs }\end{array}$ & {$[41,42]$} \\
\hline Oasis MCX & $\begin{array}{c}\text { 阳离子交换-反 } \\
\text { 相混合模式 }\end{array}$ & $\begin{array}{c}\text { 以高聚物苯乙烯/二乙烯苯为 } \\
\text { 基质键合磺酸基团具有阳离 } \\
\text { 子交换和反相吸附两种保留 } \\
\text { 机理 }\end{array}$ & 阳离子/非极性 & $1 \sim 14$ & $\begin{array}{l}\text { IAA, ABA, GA, } \\
\text { KT, CTKs, }\end{array}$ & {$[28,39,40]$} \\
\hline
\end{tabular}

处理, 通过调节氨水的不同浓度及甲醇的不同比例, 可以将不同性质的细胞分裂素从 Oasis MCX 柱上分 离, 同时, Oasis MCX 柱还适合除去有紫外吸收的干 扰杂质 ${ }^{[27]}$. 实验中发现干扰组分细胞分裂素核苷酸 可以被 $0.35 \mathrm{~mol} / \mathrm{L} \mathrm{NH}_{4} \mathrm{OH}$ 洗脱, 而游离态及核苷和 葡萄糖苷等结合态的细胞分裂素可以被 $0.35 \mathrm{~mol} / \mathrm{L}$ $\mathrm{NH}_{4} \mathrm{OH}$ 的 $60 \% \mathrm{CH}_{3} \mathrm{OH}$ 溶液洗脱.

在建立离子交换、反相、混合模式固相萃取柱的 纯化方法前, 目标植物激素的 $\mathrm{p} K_{\mathrm{a}}$ 是必需了解的参数 之一, 根据 $\mathrm{p} K_{\mathrm{a}}$ 的大小调节样品溶液的 $\mathrm{pH}$ 以确定植 物激素是以正离子、负离子或非电离状态存在以选择 合适的萃取小柱. 固相萃取柱洗脱液的酸度和有机 溶剂的比例很大程度上决定了洗脱液的洗脱能力, 需进行合理的选择和优化, 尽量满足目标化合物被 最大程度的洗脱而干扰杂质被固相萃取柱保留.

此外, 对于一些易挥发的或者经过衍生化处理 的植物激素样品, 可以选择固相吸附法进行富集浓 缩, 该方法具有操作简单、成本较低等优点. 莱莉酸 $(\mathrm{JA})$ 和水杨酸 $(\mathrm{SA})$ 是植物界广泛存在的一类重要的 信号分子, 当植物受到生物和非生物伤害后, 内源的 $\mathrm{JA}$ 或 SA 水平升高并激活植物防御反应基因的表达, 抵抗外界伤害 ${ }^{[44 ~ 47]}$. 甲酯化的茉莉酸甲酯(MeJA)和
水杨酸甲酯 $(\mathrm{MeSA})$ 能够在较低温度条件下气化, 并 被固相吸附材料所吸附, 利用这两种化合物的物理 化学性质, Engelberth 等人 ${ }^{[13]}$ 采用了一种新颖的溶剂 萃取结合固相吸附方法同时提取了植物信号分子茉 莉酸和水杨酸. Engelberth 首先使用柠檬酸水溶液: 丙 酮(30:70)作为植物激素的提取溶剂, 而 Engelberth 之所以用丙酮而没有用甲醇作为提取液是因为他认 为在甲醇提取液中 JA 和 SA 会生成相应的酯, 在溶 剂浓缩蒸发过程中造成损失, 并且丙酮的沸点 $\left(56^{\circ} \mathrm{C}\right)$ 低于甲醇 $\left(63.8^{\circ} \mathrm{C}\right)$ 的沸点更易除去. 将目标植物激素 进行甲酯化, 并加热样品瓶, 气化的 MeJA 和 MeSA 在空气流的带动下从样品中挥发而被固相吸附材料 Super Q 所吸附, 反应结束后用二氯甲烷将 MeJA 和 MeSA 从 Super Q 上洗脱, 样品可直接用气相色谱/质 谱(GC/MS)分析. 此固相吸附法一步提取, 具有操作 简单、成本较低等优点, 省略了后续的纯化过程, 并 且具有较高的回收率.

近年来发展起来的固相微萃取技术(SPME)对于 目标化合物的富集纯化只需一支类似进样器的固相 微萃取装置即可完成萃取、浓缩、解吸、进样等全部 前处理和进样工作. 目前在植物激素的提取分析中 也有应用 ${ }^{[48]}$. 在 SPME 的应用中, 要求萃取固定相的 
性质要和被分析物的性质相匹配, 使分析物在涂层 中有较快的富集和扩散速度, 能在较短时间内达到 分配平衡, 同时在进样分析时能迅速脱离固定相涂 层. 萃取固定相、提取溶剂的盐度、萃取时间、 $\mathrm{pH}$ 和温度等条件决定了样品的萃取效率. SPME 技术在 气相色谱中应用较广, 但在液相色谱中, 待测物从溶 液中被萃取到固定相上之后, 需经流动相洗脱才能 进人色谱柱中进行检测, 因此要求固定相材料必须 对流动相有足够的耐受能力, 即除了满足对待测物 有较高的萃取率外, 还能够在乙腈、甲醇等有机溶剂 中保持足够的稳定性, 而目前使用的萃取固定相较 少能完全满足这样的要求, 从而在一定程度上限制 了这一方法的应用.

固相萃取技术的应用大大简化了植物激素前处 理过程, 提高了植物激素分析的通量, 目前已经发展 成为一种成熟通用的样品处理技术. 不同填料性质 和容量大小的固相萃取柱为植物激素的前处理过程 提供了多种可供选择的样品处理方案, 以满足不同 样品的不同需要. 随着新填料的研究及新技术的推 出, 固相萃取技术在植物激素前处理过程中的应用 将日益广泛。

(iii) 免疫纯化. 免疫亲和色谱(IAC)根据抗原 与抗体之间的高亲合力、高专一性和可逆的相互作用 能够从复杂的样品基质中选择性地分离出目标分析 物, 在植物激素的纯化分析中已有应用 ${ }^{[49,50]}$. 当含有 待测物的样本粗提液经过免疫亲合色谱柱时, 提取 液中对抗体有亲合力的待测物被结合到抗体上，淋 洗除去未被结合的干扰杂质后, 再采用适当的洗脱 液将结合在抗体上的目标物洗脱下来, 从而使待测 物被选择性地提取与浓缩. 由于 IAC 是利用抗原抗 体的特异性反应来分离和纯化样品, 只有与其抗原 结构相吻合的被测物才能与抗体结合, 因此具有非 常高的选择性. 但在实际应用过程中, 由于植物激素 一般分子量相对较小, 本身不具备抗原特性, 需要与 大分子物质(载体)连接后才具备抗原特性, 在此基础 上所获取的抗体才可识别目标植物激素, 这为其特 异性抗体的制备带来困难. 在 Ulvskov 和 Nicander 的 研究中, 他们使用牛血清白蛋白作为载体分别制备 了生长素和细胞分裂素的抗体, 并已成功地应用于 植物激素的分离纯化 ${ }^{[51,52]}$, 这为 IAC 在植物激素分 析领域的应用提供了借鉴和参考. IAC 固定相中所使 用的抗体, 直接影响到目标分析物的特异性亲合力,
所以是建立 IAC 方法的关键因素. 虽然目前制备大 量性质均一、特异性识别某类植物激素的纯化抗体还 具有一定的难度，但 IAC 技术所具有的高选择性、高 灵敏度及高效能等技术特性使其在植物激素富集纯 化研究中具有广阔的发展潜力.

\section{2 分析方法}

建立植物组织中微量植物激素灵敏的测定方法, 对于阐明植物激素在植物生长发育中的作用和功能 具有十分重要的意义. 虽然植物提取物经过液液萃 取、固相萃取等手段的富集和纯化，但考虑到植物激 素在植物体内含量极低、性质不稳定，加上前处理并 不能完全消除植物组织中复杂的基质干扰, 故植物 激素的分析方法必须十分灵敏和专一，才能对植物 体内的植物激素进行准确的定量. 随着科技的发展 和创新, 越来越多的技术手段被应用于植物激素的 测定当中.

\section{1 免疫分析法}

免疫检测技术是一种以抗体作为分析试剂, 利 用抗体 $(\mathrm{Ab})$ 和抗原 $(\mathrm{Ag})$ 之间特异性相互识别结合的 特性, 对待测物进行定性或定量分析的检测方法, 主 要包括放射免疫法(RIA)和酶联免疫法(ELISA). RIA 和 ELISA 分别采用放射性同位素和酶将 $\mathrm{Ag}$ 标记成标 记抗原 $\left(\mathrm{Ag}^{*}\right)$ 与 $\mathrm{Ab}$ 结合形成 $\mathrm{Ag}^{*}-\mathrm{Ab}$ 复合物后, 再测 定其放射强度和进行酶活性定量. 其中, $\mathrm{Ag}$ 和 $\mathrm{Ab}$ 之 间反应的特异性和灵敏性是免疫检测技术的关键. 两个因素制约了这项技术在植物激素定量分析领域 的应用. 首先, 由于植物激素属于半抗原, 只有与大 分子物质(如牛血清白蛋白) 偶联制得抗原后才能刺 激动物的免疫系统而获得抗体; 其次, 植物材料提取 物中含有许多与植物激素结构相似的次生代谢物, 包括植物激素的同系物、前体、类似物以及其他性质 不明的化合物. 它们不可避免地会与抗体出现交叉 反应, 使免疫分析法的特异性降低, 影响到定量分析 结果的准确性. 所以免疫学测定法应用于植物激素 的检测直到 20 世纪 70 年代初才发展起来. 这种方法 应用于植物激素测定的最大优势在于其具有很低的 检测限和非常高的灵敏度 ${ }^{[53 \sim 55]}$.

放射免疫法(RIA)具有较高的灵敏度, 可以检测 出 $\mathrm{nmol}$ 或 $\mathrm{pmol}$ 的微量物质 ${ }^{[56,57]}$, 但需要使用 ${ }^{3} \mathrm{H},{ }^{14} \mathrm{C}$ 和 ${ }^{125} \mathrm{I}$ 等放射性同位素, 在操作时要遵守严格的操作 
规范. Weiler ${ }^{[58]}$ 制备的玉米素核苷(tZR)抗血清对玉米 素(tZ)和玉米素核苷 (tZR)都具有很高的特异性和灵 敏度, 对 IPR, cZR 和 DHZ 的交叉反应很小, 对腺苷 及相关嘌呤类化合物完全没有交叉反应. 但由于放 射性元素的不稳定性及对操作者身体的损伤, RIA 法 现已较少使用.

酶联免疫测定法(ELISA)是用某些酶对免疫原进 行标记，而避免了使用放射性物质. Wang 等人 ${ }^{[59]}$ 首 次报道了专一识别水杨酸的单克隆抗体的制备. 该 抗体原以 5-氨基水杨酸 (5-ASA)的 5 位氨基为偶联位 点, 利用血蓝蛋白 $(\mathrm{KLH})$ 为载体合成的免疫原对 $\mathrm{SA}$ 和 5-ASA 具有高亲和力. Maldiney 等人 ${ }^{[53]}$ 在测定番 茄样品中的 ZR 含量时将生物素-亲和素体系引人 ELISA 法, 可以检测出 $100 \mathrm{mg}$ 样品中的 ZR 含量, 具 有很高的灵敏度. 但是, 由于植物提取液中往往含有 较多的对植物激素测定有严重干扰的杂质, 使 ELISA 测定结果的重复性较差. 目前, 此方法在部分 植物体内含量极低的植物激素(例如油菜素等)的定 量分析中仍有应用 ${ }^{[60]}$. 此外, 免疫传感器也被用于 植物激素的测定, 主要利用抗原和抗体间的相互作 用进行识别, 当被分析物(抗原)与抗体结合后, 由转 换器将生物. 化学信号转换成电信号, 从而进行定量 检测. 湖南农业大学植物激素重点实验室在研制植 物激素免疫传感器方面取得了一定进展 ${ }^{[61,62]}$.

免疫分析法具有高选择性、灵敏度和高通量等特 点, 实验中所需样品及试剂量少, 操作简便, 但免疫 分析法不能同时对多种植物激素进行定量分析, 并 且具有不可避免的交叉反应, 影响了定量分析结果 的准确性和重现性. 这些局限性导致其在植物激素 的定性定量研究中未能得到广泛的应用.

\section{2 电化学分析法}

电化学分析法相对于气相色谱 $(\mathrm{GC})$ 、液相色谱 (HPLC)、毛细管电泳 $(C E)$ 、免疫法等分析方法具有 简单、方便和仪器价廉等特点. 在早期的研究中, 植 物激素的电化学分析方法主要是针对脱落酸 ${ }^{[63]}$ 、赤 霉素 ${ }^{[64]}$ 、玉米素和激动素 ${ }^{[65]}$ 等植物激素标准品的电 化学行为进行探讨. 通过研究发现, 植物激素存在的 本底溶液的性质和 $\mathrm{pH}$ 对测定结果会产生很大影响, 在植物样品中情况更为复杂, 所以电化学方法较少 用于实际样品的测定. 近年来, 新发展的电化学生物 传感器由于具有能在浑浊溶液中操作、选择性好、灵
敏度高等独特的优势，在植物激素的分析检测应用 中表现出良好的发展潜力.

电化学生物传感器利用具有生物活性的物质作 为识别元件(识别元件所用的生物活性物质主要有 酶、微生物、动植物组织、抗体和核酸等), 通过特 定反应使被测成分消耗或产生相应化学计量数的电 活性物质，电极上流过的电流或电极表面与溶液的 电势差会随之发生变化, 从而实现了特定物质的检 测. 李春香等人 ${ }^{[66]}$ 提出了一种以绿豆芽叶片组织-二 茂铁修饰的碳糊电极 (LFMCE) 作为测定植物激素 IAA 的组织生物传感器. 其基本原理是绿豆芽叶片 组织内含有一定量的 IAA 氧化酶, 能够催化 IAA 的 氧化代谢, 而存在于植物组织内的 IAA 氧化酶因原 有生理环境, 稳定性较高, 可用作修饰电极的敏感材 料, 利用此修饰电极的氧化峰峰电流的升高来定量 测定 IAA，其中二茂铁起了电子传递媒介体的作用。 除了修饰电极外, $\mathrm{Li}$ 等人 ${ }^{[67]}$ 开发的压电生物传感器 也可用于 IAA 的测定, 压电生物传感器是基于石英 晶体的压电效应对其电极表面质量变化进行测量的 仪器. 其基本原理是石英晶体微天平 $(\mathrm{QCM})$ 表面包 被一种抗体, 一旦样品中相应的抗原与之发生特异 性结合, 晶体的质量增加, 振荡频率发生变化, 而频 率的变化与待测抗原的浓度成正比. 在实验中，可利 用半抗原 IAA 和抗原 IAA-BSA(牛血清蛋白)与固定 在石英晶体微天平上的抗体发生竞争的免疫反应对 目标植物激素 IAA 进行检测.

近年来, 受到生物科学、信息科学和材料科学发 展成果的推动, 生物传感器技术飞速发展. 其具有灵 敏度高、检测速度快、操作简便、可进行连续动态监 测等优点, 但生物传感器的应用也同时受到稳定性、重 现性和使用寿命等诸多因素的限制, 在植物激素分 析应用领域中一直停留在方法学研究的阶段.

\section{3 色谱和色谱质谱联用}

色谱法是根据不同物质在介质中分配系数的不 同对目标化合物进行分离测定的方法.气相色谱 (GC)和高效液相色谱(HPLC)是植物激素分析领域中 较为常见的色谱分析手段. 除此之外, 依据样品中各 组分在电场作用下淌度和分配行为上的差异而实现 分离的毛细管电泳(CE) 分析方法也被归为色谱方法, 并应用于植物激素分析 ${ }^{[68,69]}$. 由于经过纯化富集处 理的植物提取物中成分依然非常复杂, 色谱分离手 
段为植物提取物提供了进一步的分离能力, 利用不 同性能的色谱检测器, 对其中的植物激素组分进行 定性和定量分析. GC 和 HPLC 在 20 世纪 70 年初开 始应用到植物激素测定后, 迅速发展成为目前植物 激素测定最为常用的测试手段. 其中, GC 法需要被 分析物具有较低的极性和气化温度, 而植物激素的 挥发性通常较差, 除了乙烯以外, 植物激素一般都需 经过衍生化处理后生成易挥发的衍生物才能进行 GC 分析, 衍生化过程较为繁琐, 增加了样品前处理的工 作量. 与之相比, HPLC 能够直接分析极性较大的植 物激素, 大大简化样品的前处理过程, 但其分离能力 较 GC 差很多. 液相色谱紫外检测器 $(U V)^{[70,71]}$ 的灵敏 度无法满足低含量植物激素的分析要求, 在植物激 素分析领域已应用较少; 而苂光检测器(FLD)则具有 很高的灵敏度和良好的选择性, 灵敏度要比紫外检 测器高 2 3 个数量级, 所需样品量较少, 比较适合部 分植物激素的测定, 在植物激素分析研究中有较多 应用报道 ${ }^{[21,22,72,73]}$. 色谱在早期的植物激素分析工作 中发挥了较大的作用, 但色谱仅根据保留时间定性, 在复杂植物提取物中可能因为不同物质保留时间相 同或相近而给出错误的结论, 紫外和苂光检测器不 能满足超低含量植物激素定性定量分析的要求、色谱 质谱联用技术在很大程度上克服了传统色谱技术在 植物激素定性和定量分析方面的不足, 成为普遍接 受和认可的植物激素分析手段.

质谱分析是一种测量离子质荷比 $(\mathrm{m} / \mathrm{z})$ 的分析方 法, 其基本原理是使样品中各组分在离子源中发生 电离, 生成不同质荷比的带电荷离子, 经加速电场的 作用, 形成离子束, 进人质量分析器进行分析. 植物 样品尽管经过多步的分离纯化和富集, 但由于激素 的超微含量和植物本底杂质的严重干扰, 因此使用 质谱对目标物进行分析, 可以使其选择性得到极大
增强. 在植物激素分析中, GC 和 HPLC 与单级四极杆 质谱仪联用技术最早得到应用, 采用选择离子监控 模式(SIM)对目标植物激素进行定量分析, 质谱在此 工作模式下, 只允许设定质荷比的植物激素分子离 子或碎片离子通过并被检测, 具有很高的灵敏度. 在 气相色谱/质谱联用 $(\mathrm{GC} / \mathrm{MS})$ 中, 化学电离源 $(\mathrm{CI})^{[13,74]}$ 和电子轰击离子源 $(\mathrm{EI})^{[16,75]}$ 是应用最广的两种电离方 式, 都曾被应用于植物激素的测定. 但是, EI 源中使 用的 $70 \mathrm{eV}$ 电子具有很高能量, 大多数化合物在此条 件下往往会发生解离, 产生大量的碎片离子, 使得定 性难度加大 ${ }^{[76]}$. 大气压电离(API)技术的出现大大扩 展了色谱质谱联用技术的适用范围, 常见的大气压 电离技术主要有电喷雾电离(ESI)和大气压化学电离 (APCI), 它克服了 $\mathrm{EI}$ 源的不足, 可通过软电离获取 目标化合物的准分子离子, 是目前最为成熟的液相 色谱/质谱 $(\mathrm{LC} / \mathrm{MS}$ ) 接口. 在软电离离子化技术中, 尽管快原子轰击 $(\mathrm{FAB})$ 离子源在植物激素测定中的 应用也有报道 ${ }^{[77]}$, 但 ESI 源具有更高的灵敏度和更 低的背景噪音 ${ }^{[78]}$. 对于一些植物激素而言, 即使选 择 SIM 工作方式, 复杂的基质仍然会干扰目标植物 激素的定量分析. 串联质谱(MS/MS)利用时间串联 的离子阱质谱(ion trap, IT)或者空间串联的三级四极 杆(QQQ)质谱实现, 在植物激素定量分析中通常使用 多级反应监控模式(multiple reaction monitor, MRM). 其工作原理如图 1 所示, 待测物在离子源形成离子, 第一级质谱分离待测物离子(母离子), 使其进入碰撞 区与惰性气体碰撞, 经过碰撞诱导解离(CID)产生子 离子, 子离子在第二级质谱内分离. 不同物质的离子 裂解规律不同, 若前体离子可能受其他物质干扰, 子 离子仍受干扰的可能性则会降低, 因而 MRM 具有比 单级质谱 SIM 工作模式更高的选择性和特异性, 可 以快速灵敏地从少量植物粗提物中直接检测出相关

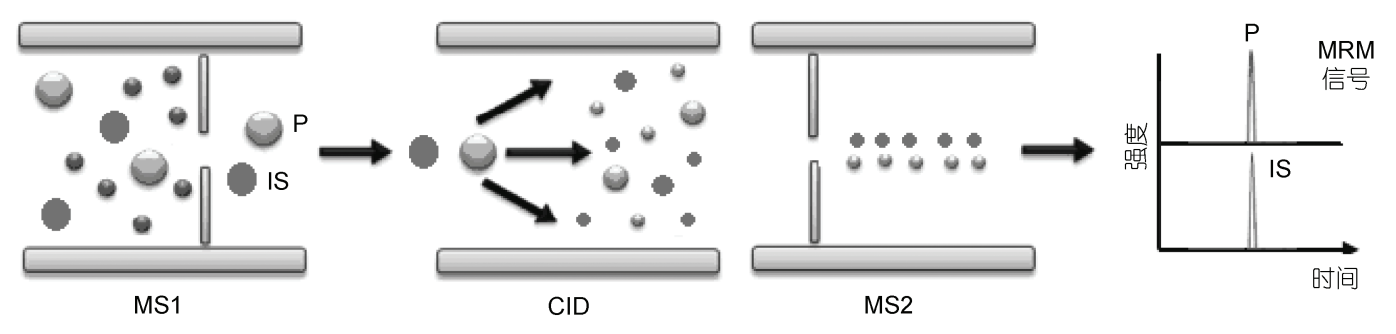

图 1 三级四极杆质谱多反应监测扫描(MRM)同位素稀释法工作原理示意图 $\mathrm{MS} 1$, 监测母离子; CID, 碰撞池; MS2, 监测子离子; IS, 同位素内标; P, 目标植物激素 


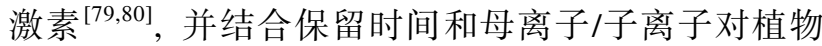
样品中的激素进行准确定性与定量. 基于 LC-MS/ MS 技术的优势，这项技术在植物激素分析中得到广 泛应用 ${ }^{[8,12,37,80]}$. 质谱技术的高灵敏极大减少了测定 样品的用量, Müller 等人 ${ }^{[81]}$ 使用 GC-MS/ MS 检测出 $20 \mathrm{mg}$ 植物样品中的 SA, JA, IAA 和 ABA 等植物激 素, 使一些珍贵的植物突变体材料内源激素的测定 变得简单易行，一步完成了多种植物激素的同时检 测。纳流电喷雾 (nanoESI) 是一种新近发展的电喷雾 离子源技术，使用纳升流速 $(1 \mathrm{~nL} / \mathrm{min} \sim 1 \mu \mathrm{L} / \mathrm{min})$ 和纳 米柱能够大幅提高系统的灵敏度, 这项技术在植物 激素的定量分析中也得到应用 ${ }^{[82]}$, 但其喷雾器喷口 容易受到样品颗粒和沉淀物的堵塞, 分析结果的稳 定性和可重现性较常规电喷雾离子源差, 并且色谱 匹配技术也有待完善, 这些原因阻碍了纳流电喷雾 技术的普及使用 ${ }^{[83]}$.

除了质谱技术的广泛使用和改进外，新的色谱 技术也逐渐应用到植物激素分析领域，近年来发展 起来的超高效液相色谱(UPLC)使用更小粒径 (1.7 $\mu \mathrm{m})$ 填料的色谱柱进行分离, 可耐受 1000 bar (1 $\mathrm{bar}=10^{5} \mathrm{~Pa}$ 的压力, 具有更强的分离能力和更快的分 析速度. UPLC 与 MS 的完美结合极大提高了仪器的 工作效率，在 HPLC-MS 上需要几十分钟完成的工作 现在仅需几分钟就可以完成, 其强大的分离效率和 分离速度为高通量检测多种植物激素提供了保障. Novak 等人 ${ }^{[10]}$ 建立了 UPLC-MS/MS(QQQ)检测 32 种 细胞分裂素的方法, 比以往建立的 HPLC-MS 方法分 析速度提高了 4 倍, 方法的精密度大幅提高. 由于 UPLC 的分离速度优势, 它可以很好地与飞行时间质 谱(UPLC-TOF MS)实现联用, 在植物激素的分析中 也有报道 ${ }^{[84]}$.

植物激素分析中有两个关键问题需要注意. 首 先，确保植物激素分析结果的准确性. 色谱质谱联用 技术充分利用质谱的工作特点, 在实际植物样品分 析中采用同位素稀释法(isotope dilution)确保分析结 果的准确性. 质谱同位素稀释法是一种灵敏度高、准 确性好的定量方法, 是在样品处理前定量加人稳定 同位素 $\left({ }^{2} \mathrm{H}\right.$ 或者 $\left.{ }^{13} \mathrm{C}\right)$ 标记的待测植物激素, 利用质谱 SIM 或者 MRM工作模式检测植物提取物中同位素内 标与内源性植物激素比率的变化来进行定量. 稳定 同位素标记的植物激素内标物的使用校正了样品在 提取和纯化过程中带来的损失及定量过程中带来的
误差, 使测定结果更为准确可信 ${ }^{[85]}$. 稳定同位素内 标与对应的植物激素几乎有着完全相同的物理化学 性质, 是理想的内标化合物, 在 MS 分析中植物激素 的稳定性同位素内标(如 ${ }^{2} \mathrm{H}_{2}$-IAA, ${ }^{2} \mathrm{H}_{6^{-}} \mathrm{ABA},{ }^{2} \mathrm{H}_{5}$-JA 和 ${ }^{2} \mathrm{H}_{4}$-SA 等)在植物激素定量分析中被广泛使用 ${ }^{[8,12,37,80]}$. 其次, 需要提高植物激素的检测灵敏度. 由于植物激 素在植物体内含量很低, 虽然经过液相萃取、固相萃 取等多种手段的分离和纯化, 部分植物激素仍然难 以进行定量, 为了提高灵敏度, 需要对目标植物激素 进行衍生化处理. 对植物激素样品衍生化处理在 GC/MS 和 LC/MS 的方法研究中也是一种重要的技术 手段. 因为植物激素的挥发性较差, 对于 GC/MS 分析, 一般需衍生化反应生成易挥发的衍生物才可以进行 分析, 已经成为气相色谱分析不可缺少的重要环节; 而植物激素提取物尽管可以直接在 LC/MS 上得到检 测，但通过合适的衍生化可将目标化合物的检测灵 敏度提高 1 3 个数量级, 能够使用更少的植物样品进 行植物激素定量分析, 因此也越来越受到研究者的 重视，在植物激素的检测分析中已有应用 ${ }^{[86 ~ 88]}$.

重氮甲烷是一种气相色谱较常用的衍生化试剂, 具有反应速率快，副产物少等优点，但其性质不稳 定，具有毒性和爆炸性，使用时必须十分小心，避免 接触皮肤并保持良好的通风 ${ }^{[89]}$. Prinsen 等人 ${ }^{[90]}$ 建立 了一个新颖的样品处理方法，他们使用重氮甲烷作 为 LC/MS 分析的衍生化试剂衍生处理了生长素 (IAA)及其代谢产物使其检测限提高了 1000 倍. 三甲

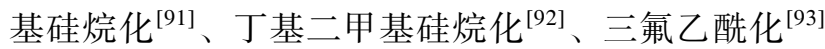
和乙酰化 ${ }^{[75]}$ 等多种衍生化方法在植物激素的 GC/MS 分析中都被使用. Björkman 和 Tillberg ${ }^{[75]}$ 采用乙酸酮 对细胞分裂素类激素进行衍生化反应后 GC/MS 检测, 具有回收率高、背景低、副产物少等优点. 但作者也 指出了此方法的缺点: $\mathrm{R}_{3}$ 为葡萄糖基的乙酰化产物 在 GC/MS 中不稳定; 乙酰化的玉米素和二氢玉米素 在 EIM 谱图中分子离子峰较弱, 因此该方法不适合 这两种类型的 CTKs 新型化合物的鉴定. Svatoš 等 人 ${ }^{[86]}$ 采用衍生化试剂丹磺酰基-3-氨基苯基硼酸与油 菜素内酯(BRs)的 22 和 23 位上的邻羟基反应生成丹 磺酰基-3-氨基苯基硼酸盐可以极大地降低检测限. 衍生化后的 BRs 的检测线可以低至 125 attomole, 从 而建立了一个 LC/MS 分析测定植物提取物中 BRs 的 高选择性和高灵敏度的方法. 因此, 对于 GC/MS 分 析, 植物激素的衍生化处理是为了改善样品挥发性, 
使其成为易于气化的样品; 而对于 LC/MS 分析, 衍 生化处理是为了提高植物激素的离子化效率, 极大 降低其检测限. 衍生化处理不同的植物激素需采用 不同的方法(表 3), 具体使用哪种衍生化试剂与所分 析的样品和衍生化效率及衍生化产物的稳定性、物理 化学性质、沸点、分子极性都有很大关系. 这些因素 是样品能否成功地使用色谱分析的先决条件, 往往 要对衍生化试剂、反应的时间和温度等条件进行多步 优化才能确定最佳方案.

\section{3 结论与展望}

植物激素在植物体内的超微含量和大量代谢干 扰物的存在使植物激素的提取和测定变得非常困难. 目前所采取的每一种提取和测定方法都有各自的选 择性和特异性, 适用于某种样品的检测. 怎样有效地
使检测灵敏度得到提高, 一直是困扰植物激素研究 者的问题之一, 也是解决低含量植物激素定量分析 的关键问题之一. 植物样品一般都需要选择合适的 纯化富集方法进行处理, 以期将干扰杂质的含量降 到最低, 才能进一步检测分析. RIA 和 ELISA 等免疫 检测法需继续完善, 减少交叉反应, 提高测定结果的 准确性. 电化学分析法受到稳定性、重现性和使用寿 命等诸多方面的限制在植物激素的检测分析中一直 未能普及应用. $\mathrm{CE}, \mathrm{GC}$ 和 $\mathrm{LC}$ 与 MS 的联用在植物激 素的检测中表现出广阔的应用前景, 特别是 LC, GC 与 $\mathrm{MS} / \mathrm{MS}$ 的联用, 使得检测灵敏度得到大幅提高, 不但可以根据离子的碎片提供重要的结构信息, 而 且因为稳定同位素内标的使用使测试结果更为准确 可靠. 其中 LC-MS/MS 联用避免了 GC-MS 分析中对 样品进行繁琐衍生化的处理过程, 已经在植物激素

表 3 植物激素的衍生化方法 ${ }^{\text {a) }}$

\begin{tabular}{|c|c|c|c|}
\hline 植物激素 & 衍生试剂 & 衍生条件 & 参考文献 \\
\hline \multirow{6}{*}{ IAA } & \multirow[t]{3}{*}{ 重氮甲烷 } & 处理后的样品溶液直接用重氮甲烷气体吹干进行反应 & {$[30]$} \\
\hline & & $10 \%$ 甲醇的二乙基醚溶液中 $0^{\circ} \mathrm{C}$ 反应 $10 \mathrm{~min}$ & [7] \\
\hline & & 甲醇溶液中在室温下快速反应 & {$[81,90]$} \\
\hline & \multirow{3}{*}{$\begin{array}{c}\text { MTBSTFA } \\
\text { BETAB }\end{array}$} & $100^{\circ} \mathrm{C}$ 反应 $60 \mathrm{~min}$ & [94] \\
\hline & & 水:三乙胺 $(25: 1$ )溶液中加人 $500 \mathrm{mmol} / \mathrm{L} \mathrm{BETAB}$ (溶于 $70 \%$ 乙腈) & {$[87]$} \\
\hline & & $80^{\circ} \mathrm{C}$ 反应 $130 \mathrm{~min}$ & \\
\hline \multirow{5}{*}{$\mathrm{ABA}$} & \multirow{2}{*}{ 重氮甲烷 } & $10 \%$ 甲醇的二乙基醚溶液中 $0^{\circ} \mathrm{C}$ 反应 $10 \mathrm{~min}$ & [7] \\
\hline & & 甲醇溶液中在室温下快速反应 & [81] \\
\hline & \multirow{3}{*}{$\begin{array}{c}\text { MTBSTFA } \\
\text { BETAB }\end{array}$} & $100^{\circ} \mathrm{C}$ 反应 $60 \mathrm{~min}$ & [94] \\
\hline & & 水:三乙胺 $(25: 1$ )溶液中加人 $500 \mathrm{mmol} / \mathrm{L}$ BETAB (溶于 $70 \%$ 乙腈) & [87] \\
\hline & & $80^{\circ} \mathrm{C}$ 反应 $130 \mathrm{~min}$ & \\
\hline \multirow{2}{*}{ JA } & MTBSTFA & $100^{\circ} \mathrm{C}$ 反应 $60 \mathrm{~min}$ & [94] \\
\hline & 重氮甲烷 & 甲醇溶液中在室温下快速反应 & [81] \\
\hline \multirow{2}{*}{ SA } & MTBSTFA & $100^{\circ} \mathrm{C}$ 反应 $60 \mathrm{~min}$ & [94] \\
\hline & 重氮甲烷 & 甲醇溶液中在室温下快速反应 & [81] \\
\hline \multirow{8}{*}{ CTKs } & \multirow{6}{*}{$\begin{array}{c}\text { MTBSTFA } \\
\text { 乙腈:乙酸酐:NMIM }(100: 10: 1) \\
\text { MSHFBA } \\
\text { BTMSA } \\
\text { 丙酸酐 }\end{array}$} & $100^{\circ} \mathrm{C}$ 反应 $60 \mathrm{~min}$ & [94] \\
\hline & & 室温下反应 $30 \mathrm{~min}$ & [75] \\
\hline & & & \\
\hline & & $90 \mathrm{C}$ 反业 $30 \mathrm{~min}$ 。 & [94] \\
\hline & & 乙腈溶液中 $60^{\circ} \mathrm{C}$ 反应 $5 \mathrm{~min}$ & [91] \\
\hline & & 乙腈: N-甲基咪唑:丙酸酐(10:6:3)溶液中 $37^{\circ} \mathrm{C}$ 反应 $30 \mathrm{~min}$ & [88] \\
\hline & \multirow[t]{2}{*}{ BETAB } & 水:三乙胺 $(25: 1$ )溶液中加人 $500 \mathrm{mmol} / \mathrm{L}$ BETAB(溶于 $70 \%$ 乙腈) & [87] \\
\hline & & $80^{\circ} \mathrm{C}$ 反应 $130 \mathrm{~min}$ & \\
\hline \multirow{4}{*}{$\begin{array}{c}\mathrm{GA}_{1}, \mathrm{GA}_{3} \\
\mathrm{GAs}\end{array}$} & \multirow{2}{*}{ BSTFA } & 重氮甲烷甲基化后加人 BSTFA 在吡啶溶液中 $80^{\circ} \mathrm{C}$ 反应 $15 \mathrm{~min}$ & [18] \\
\hline & & $90^{\circ} \mathrm{C}$ 反应 $30 \mathrm{~min}$ & [94] \\
\hline & \multirow[b]{2}{*}{ ВETAB } & 水:三乙胺 $(25: 1$ )溶液中加人 $500 \mathrm{mmol} / \mathrm{L}$ BETAB(溶于 $70 \%$ 乙腈) & [87] \\
\hline & & $80^{\circ} \mathrm{C} 反$ 应 $130 \mathrm{~min}$ & \\
\hline BRs & 丹磺酰基-3-氨基苯基硣酸 & 在嘧啶: 乙腈 $(1: 19)$ 溶液中 $62^{\circ} \mathrm{C}$ 反应 $30 \mathrm{~min}$ & [86] \\
\hline
\end{tabular}

a) MTBSTFA，N-甲基-N-(特丁基二甲基硅烷)三氟乙酰胺; MSHFBA，N-甲基-N-(三甲基硅烷)七氟丁酰胺; BSTFA，N,O-双(三

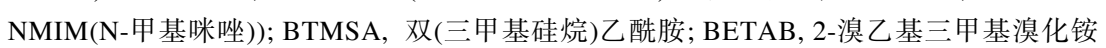


研究中发挥出巨大的作用. 与此同时, 串联质谱仪的 昂贵和同位素内标化合物的不易获得也在一方面制约 了该方法的普及推广，但色谱质谱联用技术目前依 然是植物激素检测分析中应用最为理想的技术手段.

植物激素成分及超微定量的准确检测可以帮助 研究者对植物激素调控植物生长、发育、衰老、器官 和性状形成及对环境适应等机制的深人认识，为农 作物产量和品质调控以及育种创新提供重要的理论 基础. 目前, 我国植物激素定量分析水平随着国家自 然科学基金委员会植物激素重大研究计划的启动发 展很快, 基于色谱质谱联用技术的植物激素分析平 台已经能够基本解决含量水平较高植物激素的定量 分析. 对于植物激素检测分析技术的发展趋势应包 括以下几方面: 第一, 植物激素信号传导途径一直是 生物学领域的研究热点, 并且许多主要植物激素 (生 长素、脱落酸、细胞分裂素、赤霉素等)的受体已经 得到确认 ${ }^{[95]}$. 现在已经认识到调节植物的某一生长 或生理反应过程是由多种激素协同作用完成的 ${ }^{[96]}$, 对植物某单一激素的研究已不能真实地解释植物所 表现出的生理现象，对其起协同作用的多种激素的 同时检测才能反映出植物激素相互作用的本质. 因
此需要建立植物激素组的分析方法体系，既可同时 分析不同激素的组合(种类和含量), 又可检测特定激 素不同结合状态的分布比例或含量. 第二, 植物激素 在植物体内的不同时期、不同组织及不同器官的分布 水平不同, 建立植物激素的超微量、高灵敏、原位、瞬 时、动态的分析方法可以从单分子、单细胞以及活体 原位中分析检测植物激素, 也帮助研究者更加深人 了解植物激素的合成及代谢本质. 第三, 植物激素标 准品或同位素标记的植物激素标准品是植物激素准 确定量的基本条件，但对其提取和制备却非常困难. 因此，建立大量合成与提取植物激素或同位素标记 的植物激素标准品及其部分关键中间体的方法也是 今后研究工作者需要努力的方向. 第四, 需要建立高 通量、快速、高效分离与预富集的植物样品处理方法, 提高植物激素的检测效率, 可以对生物学研究的实 验结果有迅速的反馈. 可以预见，随着科学技术的快 速发展，植物激素的检测水平也会得到突飞猛进的 进步，新型仪器的开发和联用将在植物激素定量分 析和结构鉴定中发挥重要的作用, 并将会有越来越 多的新型植物激素的发现达到深人认识植物激素在 植物的生长发育过程中所起作用的本质.

\section{参考文献}

1 Davies P J. Plant Hormones: Physiology, Biochemistry and Molecular Biology. Dordrecht: Kluwer Academic Publishers, 1995. 1-12

2 Harman G E, Howell C R, Viterbo A, et al. Trichoderma species-opportunistic avirulent plant symbionts. Nat Rev Microbiol, 2004, 2: 43-56

3 Bray E A. Abscisic acid regulation of gene expression during water-deficit stress in the era of the Arabidopsis genome. Plant Cell Environ, 2002, 25: $153-161$

4 Durrant W E, Dong X. Systemic acquired resistance. Annu Rev Phytopathol, 2004, 42: 185-209

5 郝建军, 康宗利. 植物生理学. 北京: 化学工业出版社, 2005. 160

6 Barkawi L S, Tam Y Y, Tillman J A, et al. A high-throughput method for the quantitative analysis of indole-3-acetic acid and other auxins from plant tissue. Anal Biochem, 2008, 372: 177-188

7 Vine J H, Noiton D, Plummer J A, et al. Simultaneous quantitation of indole 3-acetic acid and abscisic acid in small samples of plant tissue by gas chromatography-mass spectrometry/selected ion monitoring. Plant Physiol, 1987, 85: 419-422

8 Ross A R S, Ambrose S J, Cutler A J, et al. Determination of endogenous and supplied deuterated abscisic acid in plant tissues by high-performance liquid chromatography-electrospray ionization tandem mass spectrometry with multiple reaction monitoring. Anal Biochem, 2004, 329: 324-333

9 Vilaró F, Canela-Xandri A, Canela R. Quantification of abscisic acid in grapevine leaf (Vitis vinifera) by isotope-dilution liquid chromatography-mass spectrometry. Anal Bioanal Chem, 2006, 386: 306-312

10 Novak O, Hauserova E, Amakorova P, et al. Cytokinin profiling in plant tissues using ultra-performance liquid chromatography-electrospray tandem mass spectrometry. Phytochemistry, 2008, 69: 2214-2224

11 Gawronska H, Yang Y Y, Furukawa K, et al. Effects of low irradiance stress on gibberellin levels in pea-seedlings. Plant Cell Physiol, 1995, 36: 1361-1367

12 Kristl J, Veber M, Krajničič B, et al. Determination of jasmonic acid in Lemna minor (L.) by liquid chromatography with fluorescence detection. Anal Bioanal Chem, 2005, 383: 886-893

13 Engelberth J, Schmelz E A, Alborn H T, et al. Simultaneous quantification of jasmonic acid and salicylic acid in plants by vapor-phase 
extraction and gas chromatography-chemical ionization-mass spectrometry. Anal Biochem, 2003, 312: 242-250

14 Cao J, Murch S J, O’Brien R, et al. Rapid method for accurate analysis of melatonin, serotonin and auxin in plant samples using liquid chromatography-tandem mass spectrometry. J Chromatogr A, 2006, 1134: 333-337

15 Liu B F, Zhong X H, Lu Y T. Analysis of plant hormones in tobacco flowers by micellar Electrokinetic capillary chromatography coupled with on-line large volume sample stacking. J Chromatogr A, 2002, 945: 257-265

16 Zhang F J, Jin Y J, Xu X Y, et al. Study on the extraction, purification and quantification of jasmonic acid, abscisic acid and indole-3-acetic acid in plants. Phytochem Anal, 2008, 19: 560-567

17 Moritz T, Olsent J E. Comparison between high resolution selected ion monitoring, selected reaction monitoring and four-sector tandem mass spectrometry quantitative analysis of gibberellins in milligram amounts of plant tissue. Anal Chem, 1995, 67: 1711-1716

18 Symons G M, Reid J B. Hormone levels and response during de-etiolation in Pea. Planta, 2003, 216: 422-431

19 Jager C E, Symons G M, Nomura T, et al. Characterization of two brassinosteroid C-6 oxidase genes in Pea. Plant Physiol, 2007, 143: $1894-1904$

20 Zullo M A T, Adam G. Brassinosteroid phytohormones-structure, bioactivity and applications. Braz J Plant Physiol, 2002, 14: 143-181

21 Rozhon W, Petutschnig E, Wrzaczek M, et al. Quantification of free and total salicylic acid in plants by solid-phase extraction and isocratic high-performance anion-exchange chromatography. Anal Bioanal Chem, 2005, 382: 1620-1627

22 Eshita S M. 3-hydroxybenzoic acid as an internal standard for the high-pressure liquid chromatography quantitation of salicylic acid in plants. Anal Biochem, 2001, 289: 99-102

23 Enyedi A J, Yalpani N, Silverman P, et al. Localization, conjugation and function of salicylic acid in tobacco during the hypersensitive reaction to tobacco mosaic virus. Proc Natl Acad Sci USA, 1992, 89: 2480-2484

24 Bieleski R L. The problem of halting enzyme action when extracting plant tissues. Anal Biochem, 1964, 9: 431-442

25 Horgan R, Scott I M. Cytokinins. In: Rivier L, Crozier A, eds. The Principles and Practice of Plant Hormone Analysis. London: Academic Press, 1987. 304-365

26 Laloue M, Terrine C, Gawer M. Cytokinins: Formation of the nucleoside-50-triphosphate in tobacco and Acer cells. FEBS Lett, 1974, 46: $45-50$

27 Hoyerová K, Gaudinová A, Malbeck J, et al. Efficiency of different methods of extraction and purification of cytokinins. Phytochemistry, 2006, 67: 1151-1159

28 Izumi Y, Okazawa A, Bamba T, et al. Development of a method for comprehensive and quantitative analysis of plant hormones by highly sensitive nanoflow liquid chromatography-electrospray ionization-ion trap mass spectrometry. Analyt Chim Acta, 2009, 648: 215-225

29 Wu Y, Hu B. Simultaneous determination of several phytohormones in natural coconut juice by hollow fiber-based liquid-liquid-liquid microextraction-high performance liquid chromatography. J Chromatogr A, 2009, 1216: 7657-7663

30 Ribnicky D M, Cooke T J, Cohen J D. A microtechnique for the analysis of free and conjugated indole-3-acetic acid in milligram amounts of plant tissue using a benchtop gas chromatograph-mass spectrometer. Planta, 1998, 204: 1-7

31 Wijayanti L, Kobayashi M, Fujioka S, et al. Identification and quantification of abscisic acid, indole-3-acetic acid and gibberllins in phloem exudates of Pharbitis nil. Biosci Biotech Biochem, 1995, 59: 1533-1535

32 Dunlap J R, Guinn G. A simple purification of indole-3-acetic acid and abscisic acid for GC-SIM-MS analysis by microfiltration of aqueous samples through Nylon. Plant Physiol, 1989, 90: 197-201

33 Varbanova M, Yamaguchi S, Yang Y, et al. Methylation of gibberellins by Arabidopsis GAMT1 and GAMT2. Plant Cell, 2007, 19: 32-45

34 Astot C, Dolezal K, Moritz T, et al. Deuterium in vivo labelling of cytokinins in Arabidopsis thaliana analysed by capillary liquid chromatography/frit-fast atom bombardment mass spectrometry. J Mass Spectrom, 2000, 35: 13-22

35 Novák O, Tarkowski P, Tarkowská D, et al. Quantitative analysis of cytokinins in plants by liquid chromatography-single-quadrupole mass spectrometry. Analyt Chim Acta, 2003, 480: 207-218

36 Ma Z, Ge L, Lee A S Y, et al. Simultaneous analysis of different classes of phytohormones in coconut (Cocos nucifera L.) water using high-performance liquid chromatography and liquid chromatography-tandem mass spectrometry after solid-phase extraction. Analyt Chim Acta, 2008, 610: 274-281

37 Matsuda F, Miyazawa H, Wakasa K, et al. Quantification of indole-3-acetic acid and amino acid conjugates in rice by liquid chromatography-electrospray ionization-tandem mass spectrometry. Biosci Biotechnol Biochem, 2005, 69: 778-783

38 Rhijn J A V, Heskamp H H, Davelaar E, et al. Quantitative determination of glycosylated and aglycon isoprenoid cytokinins at sub-picomolar levels by microcolumn liquid chromatography combined with electrospray tandem mass spectrometry. J Chromatogr A, 2001, 929: $31-42$

39 Ge L, Yong J W H, Goh N K, et al. Identification of kinetin and kinetin riboside in coconut (Cocos nucifera L.) water using a combined approach of liquid chromatography-tandem mass spectrometry, high performance liquid chromatography and capillary electrophoresis. $\mathrm{J}$ 
Chromatogr B, 2005, 829: 26-34

40 Ge L, Yong J W H, Tan S N, et al. Analysis of some cytokinins in coconut (Cocos nucifera L.) water by micellar electrokinetic capillary chromatography after solid-phase extraction. J Chromatogr A, 2004, 1048: 119-126

41 Ge L, Yong J W H, Tan S N, et al. Analysis of cytokinin nucleotides in coconut (Cocos nucifera L.) water using capillary zone electrophoresis-tandem mass spectrometry after solid-phase extraction. J Chromatogr A, 2006, 1133: 322-331

42 Dobrev P I, Havlíček L, Vágner M, et al. Purification and determination of plant hormones auxin and abscisic acid using solid phase extraction and two-dimensional high performance liquid chromatography. J Chromatogr A, 2005, 1075: 159-166

43 Hou S, Zhu J, Ding M, et al. Simultaneous determination of gibberellic acid, indole-3-acetic acid and abscisic acid in wheat extracts by solid-phase extraction and liquid chromatography-electrospray tandem mass spectrometry. Talanta, 2008, 76: 798—802

44 Smith J L, Moraes C M D, Mescher M C. Jasmonate-and salicylate-mediated plant defense responses to insect herbivores, pathogens and parasitic plants. Pest Manag Sci, 2009, 65: 497-503

45 Baldwin I T, Zhang Z P, Diab N, et al. Quantification, correlations and manipulations of wound-induced changes in jasmonic acid and nicotine in Nicotiana sylvestris. Planta, 1997, 201: 397-404

46 Chen H, Wilkerson C G, Kuchar J A, et al. Jasmonate-inducible plant enzymes degrade essential amino acids in the herbivore midgut. Proc Natl Acad Sci USA, 2005, 102: 19237-19242

47 Koo A J K, Gao X, Daniel J A, et al. A rapid wound signal activates the systemic synthesis of bioactive jasmonates in Arabidopsis. Plant J, 2009, 59: 974-986

48 Liu H T, Li Y F, Luan T G, et al. Simultaneous determination of phytohormones in plant extracts using SPME and HPLC. Chromatographia, 2007, 66: 515-520

49 Vankova R, Gaudinova A, Sussenbekova H, et al. Comparison of oriented and random antibody immobilization in immunoaffinity chromatography of cytokinins. J Chromatogr A, 1998, 811: 77-84

50 Hauserová E, Swaczynová J, Doležal K, et al. Batch immunoextraction method for efficient purification of aromatic cytokinins. J Chromatogr A, 2005, 1100: 116-125

51 Ulvskov P, Marcussen J, Rajagopal R, et al. Immunoaffinity purification of indole-3-acetamide using monoclonal antibodies. Plant Cell Physiol, 1987, 28: 937-945

52 Nicander B, Stahl U, Bjorkman P O, et al. Immunoaffinity co-purification of cytokinins and analysis by high-performance liquid chromatography with ultraviolet-spectrum detection. Planta, 1993, 189: 312-320

53 Maldiney R, Leroux B, Sabbagh I, et al. A biotin-avidin-based enzyme immunoassay to quantify three phytohormones: Auxin, abscisic acid and zeatin-riboside. J Immunol Methods, 1986, 90: 151-158

54 Morris R O, Blevins D G, Dietrich J T, et al. Cytokinins in plant pathogenic bacteria and developing cereal grains. Aust J Plant Physiol, 1993, 20: 621-637

55 Yong J W H, Wong S C, Letham D S, et al. Effects of elevated $\left[\mathrm{CO}_{2}\right]$ and nitrogen nutrition on cytokinins in the xylem sap and leaves of cotton. Plant Physiol, 2000, 124: 767-779

56 Grayling A, Hanke D E. Cytokinins in exudates from leaves and roots of red Perilla. Phytochemistry, 1992, 31: 1863-1868

57 Cook N C, Bellstedt D U, Jacobs G. Endogenous cytokinins distribution patterns at budburst in Granny Smith and Braeburn apple shoots in relation to bud growth. Sci Hort, 2001, 87: 53-63

58 Weiler E W. Radioimmunoassays for trans-zeatin and related cytokinins. Planta, 1980, 149: 155-162

59 Wang S C, Li G J, Kai X, et al. Preparation and application of monoclonal antibodies specific for salicylic acid. Acta Bot Sin, 2001, 43: $1207-1210$

60 Swaczynová J, Novák O, Hauserová E, et al. New techniques for the estimation of naturally occurring brassinosteroids. J Plant Growth Regul, 2007, 26: 1-14

61 Li J, Wu Z Y, Xiao L T, et al. A novel piezoelectric biosensor for the detection of phytohormones-indole acetic acid. Analyt Sci, 2002, 18: $1-5$

62 李春香, 李劲, 萧浪涛. 基于統基自组装单层膜的植物生长激素吲哚乙酸电化学免疫传感器的研究. 化学学报, 2003, 61: 790-794

63 王宏, 胡胜水, 周性尧. 植物激素脱落酸伏安行为的研究. 武汉大学学报, 1997, 43: 721-724

64 刘旭红, 王力生, 蒋治良. 赤霉素的 2.5 次微分伏安法研究及应用. 分析科学学报, 1997, 13: 222-224

65 江子伟, 姜䚲波, 琚常青, 等. 玉米素和激动素的伏安行为. 高等学校化学学报, 1994, 15: 355-359

66 李春香, 李劲, 萧浪涛, 等. 植物激素吲哚乙酸电化学生物传感器的研究. 分析科学学报, 2003, 19: 205一208

67 Li J, Wu Z Y, Xiao L T. A novel piezoelectric biosensor for the detection of phytohormone $\beta$-indole acetic acid. Analyt Sci, 2002, 18: 403407

68 Ge L, Peh C Y C, Yong J W H, et al. Analyses of gibberellins by capillary electrophoresis-mass spectrometry combined with solid-phase 
extraction. J Chromatogr A, 2007, 1159: 242-249

69 Ge L, Yong J W H, Tan S N, et al. Determination of cytokinins in coconut (Cocos nucifera L.) water using capillary zone electrophoresis-tandem mass spectrometry. Electrophoresis, 2006, 27: 2171-2181

70 Albrecht T, Kehlen A, Stahl K, et al. Immunoaffinity co-purification of cytokinins and analysis by high-performance liquid chromatography liquid chromatography with ultraviolet-spectrum detection. Planta, 1993, 191: 86-94

71 Gamoh K, Kitsuwa T, Takatsuto S, et al. Determination of trace brassinosteroids by high performance liquid chromatography. Analyt Sci, 1988, 4: 533-535

72 Verberne M C, Brouwer N, Delbianco F, et al. Method for the extraction of the volatile compound salicylic acid from tobacco leaf material. Phytochem Anal, 2002, 13: 45-50

73 符继红, 褚金芳, 王吉德, 等. 固相萃取反相高效液相色谱菼光检测法测定拟南芥中的生长素. 分析化学, 2009, 37: 1324一 1327

74 Schmelz E A, Engelberth J, Alborn H T, et al. Airborne signals prime plants against insect herbivore attack. Proc Natl Acad Sci USA, 2003, 100: 10552-10557

75 Björkman P O, Tillberg E. Acetylation of cytokinins and modified adenine compounds: A simple and non-destructive derivatization method for gas chromatography-mass spectrometric analysis. Phytochem Anal, 1996, 7: 57-68

76 Ikekawa N, Takatsuto S. Microanalysis of brassinosteroids in plants by gas chromatography/Mass spectrometry. Mass Spectroscopy, 1984, 32: $55-70$

77 Astot C, Dolezal K, Moritz T, et al. Precolumn derivatisation and capillary liquid chromatographic/frit-fast atom bombardment mass spectrometry analysis of cytokinins in Arabidopsis thaliana. J Mass Spectrom, 1998, 33: 892-902

78 Tarkowski P, Ge L, Yong J W H, et al. Analytical methods for cytokinins. Trends Analyt Chem, 2009, 28: 323-335

79 Pan X Q, Welti R, Wang X M. Simultaneous quantification of major phytohormones and related compounds in crude plant extracts by liquid chromatography-electrospray tandem mass spectrometry. Phytochemistry, 2008, 69: 1773-1781

80 Segarra G, Jáuregui O, Casanova E, et al. Simultaneous quantitative LC-ESI-MS/MS analyses of salicylic acid and jasmonic acid in crude extracts of Cucumis sativus under biotic stress. Phytochemistry, 2006, 67: 395-401

81 Müller A, Düchting P, Weiler E W. A multiplex GC-MS/MS technique for the sensitive and quantitative single-run analysis of acidic phytohormones and related compounds and its application to Arabidopsis thaliana. Planta, 2002, 216: 44-56

82 Izumi Y, Okazawa A, Bamba T, et al. Development of a method for comprehensive and quantitative analysis of planthormones by highly sensitive nanoflow liquid chromatography-electrospray ionization-ion trap mass spectrometry. Analyt Chim Acta, 2009, 648: 215-225

83 帕拉马尼克 B N, 甘古利 A K, 格罗斯 M L. 电喷雾质谱应用技术. 北京：化学工业出版社, 2005.132

84 Glauser G, Grata E, Dubugnon L, et al. Spatial and temporal dynamics of jasmonate synthesis and accumulation in Arabidopsis in response to wounding. J Biol Chem, 2008, 283: 16400-16407

85 Rivier L C. Principles and Practice of Plant Hormones Analysis, 1 and 2. London: Academic Press, 1987

86 Svatoš A, Antonchick A, Schneider B. Determination of brassinosteroids in the sub-femtomolar range using dansyl-3-aminophenylboronate derivatization and electrospray mass spectrometry. Rapid Commun Mass Spectrom, 2004, 18: 816-821

87 Kojima M, Kamada-Nobusada T, Komatsu H. Highly sensitive and high-throughput analysis of plant hormones using MS-Probe modification and liquid chromatography-tandem mass spectrometry: An application for hormone profiling in Oryza sativa. Plant Cell Physiol, 2009, 50: $1201-1214$

88 Ordström A, Tarkowski P, Tarkowska D, et al. Derivatization for LC-electrospray ionization-MS: A tool for improving reversed-phase separation and ESI responses of bases, ribosides, and intact nucleotides. Anal Chem, 2004, 76: 2869-2877

89 王立, 汪正范. 色谱分析样品处理. 北京: 化学工业出版社, 2006

90 Prinsen E, Dongen W V, Esmans E L, et al. HPLC linked electrospray tandem mass spectrometry: A rapid and reliable method to analyse indole-3-acetic acid metabolism in bacteria. J Mass Spectrom, 1997, 32: 12-22

91 Most B H, Williams J C, Parker K J. Gas chromatography of cytokinins. J Chromatogr A, 1968, 38: 136-138

92 Hocart C H, Wong O C, Letham D S, et al. Mass spectrometry and chromatography of t-butyldimethylsilyl derivatives of cytokinin bases. Anal Biochem, 1986, 153: 85-96

93 Ludewig M, Dörffling K, König W A. Electron-capture capillary gas chromatography and mass spectrometry of trifluoroacetylated cytokinins. J Chromatogr A, 1982, 243: 93-98

94 Birkemeyer C, Kolasa A, Kopka J. Comprehensive chemical derivatization for gas chromatography-mass spectrometry-based multi-targeted profiling of the major phytohormones. J Chromatogr A, 2003, 993: 89-102

95 Santner A, Estelle M. Recent advances and emerging trends in plant hormone signaling. Nature, 2009, 456: 1071-1078

96 Rost T L, Weier T E. Botany, An Introduction to Plant Biology. New York: Wiley, 1979. 155-170 